\title{
Nanoparticle Generation From Nitinol Target Using Pulsed Laser Ablation
}

\author{
Tomi Smausz ${ }^{*}$, Gabriella Kecskeméti* ${ }^{* *}$, Bence Kondász ${ }^{* *}$, Gergely Papp ${ }^{* *}$, Zsolt Bengery ${ }^{* *}$, Judit \\ Kopniczky** and Béla Hopp $^{* *}$ \\ "MTA-SZTE Research Group on Photoacoustic Spectroscopy, University of Szeged, H-6720 Szeged, \\ Dóm tér 9, Hungary \\ E-mail:tomi@physx.u-szeged.hu \\ ${ }^{* *}$ Department of Optics and Quantum Electronics, University of Szeged, H-6720 Szeged, \\ Dóm tér 9, Hungary
}

\begin{abstract}
The influence of experimental conditions (wavelength, liquid environment) on the properties of Ni-Ti nanoparticles generated by nanosecond laser ablation of NiTinol (Shape Memory Alloy) target and the elemental distribution of the irradiated surface were investigated. The studied laser wavelengths were $248(\mathrm{KrF})$ and $1064 \mathrm{~nm}$ (Q-switched Nd:YAG). Nitinol targets were covered with thin liquid layer of distilled water and ethanol, respectively. The samples were irradiated with various numbers of pulses (500 in case of the investigation of the alloy surface and 20000 for the particle generation) and fluences ( 2 and $5 \mathrm{~J} / \mathrm{cm} 2)$. The morphology of laser treated NiTi surface and the size of the generated nanoparticles were studied as a function of both the laser fluence and the type of the applied liquids using scanning electron microscopy (SEM). Element mappings were realized by energy-dispersive X-ray spectroscopy (EDX). Our results clearly show that when using ethanol both the laser treated surface and the generated particles had a relatively homogeneous elemental distribution. However, under distilled water the irradiated surface and the generated particles showed a separated presence of $\mathrm{Ni}$ and $\mathrm{Ti}$ elements. For the higher fluence, formation of core-shell structured particles were observed in water environment.
\end{abstract}

DOI: $10.2961 /$ jlmn.2015.02.0012

Keywords: NiTinol, Shape Memory Alloy, laser ablation, nanoparticles, nickel, titanium

\section{Introduction}

Nanoparticles have attracted great attention due to the numerous application possibilities based on the wide range of available physical, chemical and even biological (active/inert) properties. Since the properties of metal nanoparticles strongly depend on their shape and size distribution extensive studies have been done on controlling these parameters in various synthesis methods. There are different kinds of chemical and physical techniques to produce nanoparticles; among physical methods, laser ablation is a promising technique. This technique enables a nanoparticle generation of nearly unlimited variety of metals. Laser ablation of metals in liquid environment has become a powerful emerging technique with substantial scientific, industrial and medical applications [1]. The ablation process is able to produce very gentle material removal, converting a bulk material into nanoparticles in gases and liquids [2] without changing its stoichiometry [3, 4]. Laser ablation has some advantages as a production method, such as a disperse distribution of the colloids in the solvent, no use of toxic chemical precursors, chemically pure nanoparticle generation, and it is applicable for different materials. Laser ablation of solid targets in liquid medium can be used to fabricate nanostructures with various compositions and morphologies and to generate high purity nanoparticles in a variety of liquids $[5,6,7]$. In most cases the process is carried out using infrared lasers and infrared transparent liquids (such as water, ethanol, acetone, ethyl acetate, acrylates, etc.) where in-situ dispersion of the nanoparticles in these solvents is possible. When the target is a noble metal or material such as carbon or silicon, the ablated products are usually pure elemental particles, which are formed in the laser generated plasma in the absence of any chemical reactions with the liquid medium [8]. Shape memory alloys (SMA) have become increasingly interesting in recent years because of their unique properties. The most common shape memory material is an alloy of nickel and titanium called Nitinol. This particular alloy is used in industrial applications due to the low manufacturing cost, the very good electrical and mechanical properties, long fatigue life and high corrosion resistance. Earlier studies showed that the produced NiTi nanoparticles had a titanium oxide layer on the surface, giving a core-shell nature to the nanoparticles. The thickness of the layer differs, depending on the organic liquids used for suspension [9]. Core-shell nanostructures, have attracted increasing research interest due to their unique structural features that consist of an inner core and an external shell of different chemical compositions. The inherent emergent chemical and physical properties of core-shell nanostructures are of great importance to broad range of applications including electronics, magnetism, optics, and catalysis. In the last years a large number of core-shell nanostructures have been successfully fabricated using approaches ranging from laser ablation and hightemperature evaporation to carbothermal reduction and hydrothermal methods [10]. In present work we studied the 
generation of nanoparticles by laser ablation of NiTi targets in various liquid environments using different type of ns pulsed lasers with several fluences. In addition the morphology and elemental distribution of the irradiated targets were also investigated.

\section{Experimental}

The target was bulk nitinol (Fort Wayne Metals, 54.84 wt $\% \mathrm{Ni}$ and $45.14 \mathrm{wt} \% \mathrm{Ti}$ ) and ablation was carried out using pulses of a Nd:YAG laser (1064 nm, FWHM=8 ns) and an excimer $\mathrm{KrF}$ laser (248 nm, FWHM=18 ns) while the target was immersed in distilled water and ethanol, respectively. The applied experimental setup for laser ablation is schematically illustrated in Fig. 1.

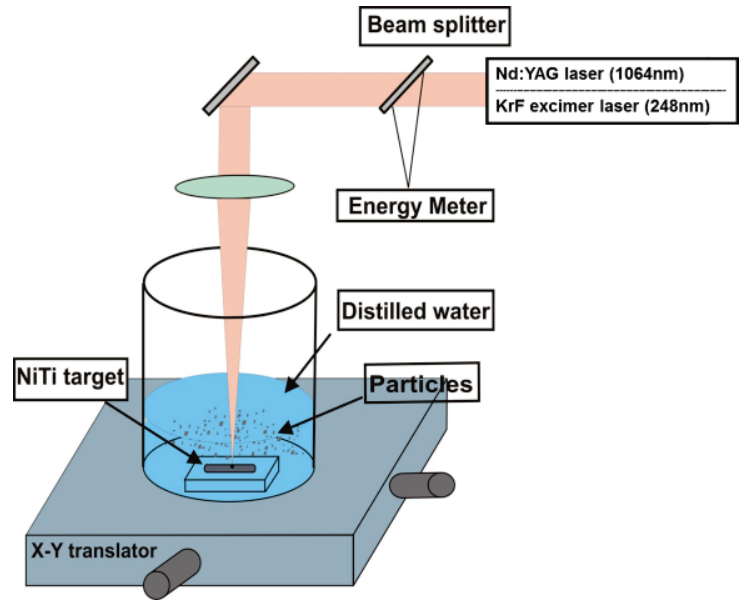

Figure 1. The applied experimental setup used for nanoparticle generation. A high intensity laser pulse ( $\mathrm{KrF}, \mathrm{Nd}: \mathrm{YAG})$ is focused onto NiTi target, under liquid.

The laser beam was focused onto the metal wire through the $2 \mathrm{~mm}$ thick liquid layer onto a $0.6 \mathrm{~mm}^{2}$ area. The samples were irradiated with 500 pulses in case of the investigation of the alloy surface and 25000 pulses for the particle generation. The laser fluence was 2 and $5 \mathrm{~J} / \mathrm{cm}^{2}$, respectively, and the repetition rate was $5 \mathrm{~Hz}$. During the ablation process the target was translated in order to avoid large crater formation on the target surface. The generated nanoparticles formed a colloidal solution with the liquid that surrounds the target. The morphology of the nanoparticles was studied by SEM (Hitachi S4700), they chemical composition mapping were realized by energydispersive X-ray spectroscopy (EDX).

\section{Results and discussion}

The morphology of the irradiated targets was investigated with SEM, and was compared to the elemental distribution map of $\mathrm{Ni}$ and $\mathrm{Ti}$ recorded with EDX of the very same area, as shown in Fig. 2. The elements are indicated with red (Ni) and green (Ti) colors in the EDX images. These images show the surface morphology of the untreated reference NiTi (Fig. 2 A,D) and targets irradiated with 500 pulses of $2 \mathrm{~J} / \mathrm{cm}^{2}$ in distilled water and ethanol using the two different laser types. The Nd:YAG treated surface (Fig. 2 C,F) shows evidence of intensive melting and boiling and contains several round-shaped, attached particles. In comparison, the $\mathrm{KrF}$ treated surface (Fig. 2 $\mathrm{B}, \mathrm{E})$. remained relatively smooth. In case of distilled water the elemental maps show a separated presence of $\mathrm{Ni}$ and $\mathrm{Ti}$ elements. This fact indicates that during melting, evaporation and resolidification of the target material, a disgregation of the components occurs. When using ethanol the distribution remains uniform all over the treated area with an apparent Ti enrichment on the surface in case of $\mathrm{Nd}$ :YAG laser.

After each experiment, the obtained colloidal suspensions were dropped on Si plates for examination of particle morphology and microstructure. After the evaporation of the distilled water and ethanol the generated particles attached to the plates were investigated by SEM. Since the nanoparticles generated from the bulk alloy might have been disproportioned during laser ablation, element mapping was carried out using EDX of the created nanoparticle samples. The element distribution of nanoparticles generated in water and ethanol are shown in Fig. 3. It can be seen that the samples contain a reach variety of nanoparticles and clusters. The ablated particles in water and in ethanol produced stable colloids of NiTi particles. The observed morphology of the particles was almost a perfect sphere, and in all experiments their size is comprised between 50 and $1000 \mathrm{~nm}$ and their average size is around $200 \mathrm{~nm}$. In distilled water a significant aggregation of the nanoparticles was observed a few days after preparation. The results of the EDX measurements on nanoparticles were in accordance with those observed for the irradiated surfaces. In case of ethanol the produced nanoparticles had a uniform elemental distribution while in case of water element separation was observed. At the applied lower fluence $\left(2 \mathrm{~J} / \mathrm{cm}^{2}\right)$ many of the spheres were either nickel or titanium dominated, others showed a more balanced composition. The size and the morphology of the

\section{Untreated}

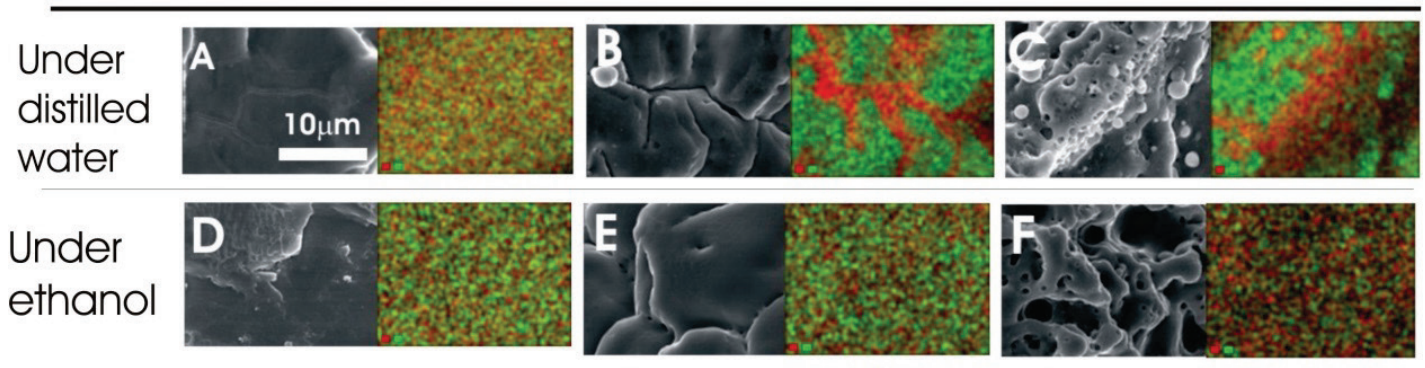

Figure 2. SEM and EDX images showing the untreated NiTi target (A,D) and its surface after laser irradiation with $2 \mathrm{~J} / \mathrm{cm}^{2}$ fluence using $\mathrm{KrF}$ excimer $(\mathrm{B}, \mathrm{E})$ and $\mathrm{Nd}$ :YAG $(\mathrm{C}, \mathrm{F})$ lasers under distilled water and ethanol. 


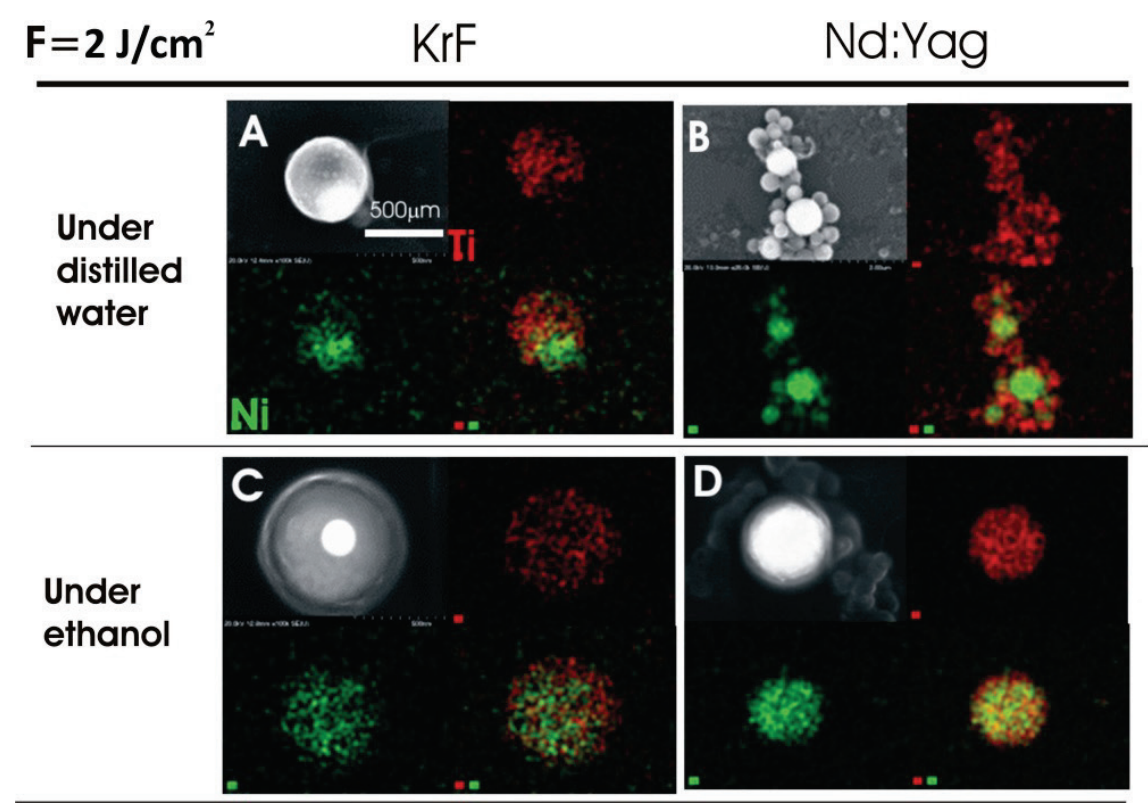

Figure 3. SEM image of the generated particles and clusters and EDX mapping images of the same area indicating the distribution of $\mathrm{Ni}$ (green) and $\mathrm{Ti}$ (red) elements.

$\mathrm{F}=5 \mathrm{~J} / \mathrm{cm}^{2} \quad$ Under distilled water

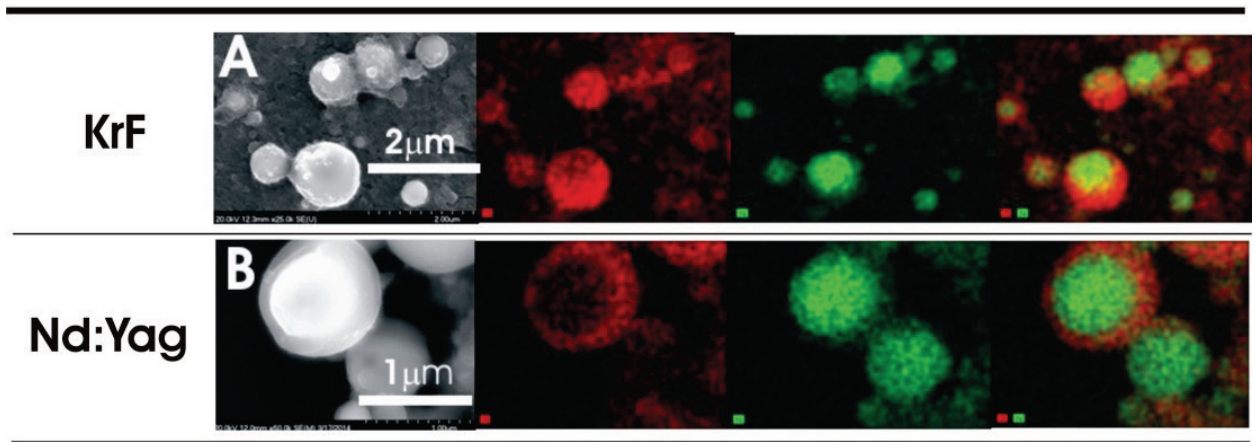

Figure 4. Example of SEM images and elemental distribution maps showing core(Ni)shell(Ti) nanoparticle structures.

particles are governed by laser parameters and the surrounding liquid. The interaction between the laser beam and the NiTi target submerged in solvent leads to heating up a very thin layer of metal above its melting point. Due to heat transfer from the metal to liquid, the temperature of the adjacent solvent layer also increases and evaporation can occur. The interaction of the metal with the oxygen content from the vaporized solvent results in the formation of titanium oxide. As shown earlier in various studies, the pulsed laser ablation of pure titanium targets in water results in the formation of $\mathrm{TiO}_{\mathrm{x}}$ nanoparticles [11]. This is an important point to take into account in the formation mechanism, because it is strongly related to the fabrication process. Some authors reported the formation of nanoparticles containing exclusively metallic titanium, others also have obtained particles with a range of oxygen content by ablating a titanium target submerged in water with a pulsed Nd:YAG laser. In ethanol similar results could be obtained with use of continuous wave lasers which allow a longer interaction between the evaporated metal and the components of the evaporated/decomposed liquid [12]. In presence of $\mathrm{OH}^{-}$group the oxidation of Ti is much faster as compared to pure oxygen. Studies showed that during the high temperature oxidation of bulk NiTi a nickel-free oxidized zone was formed with a relatively small amount of $\mathrm{Ni}$ species at the air/oxide interface, while most of the nickel content accumulated under the oxidized zone [13]. This can give a good explanation for the formation of core-shell structure observed in case of larger $(>1 \mu \mathrm{m})$ nanoparticles which appeared at the higher (5 $\mathrm{J} / \mathrm{cm}^{2}$ ) fluence (Fig. 4.). These particles have a nickel core and a titanium enriched coating, which is possibly titanium-oxide. Unfortunately, our EDX device was unable to give reliable information on the distribution of oxygen in the samples. Further analysis is required for determining whether the core of such particles is purely nickel or the desired nickel-titanium alloy. 


\section{Conclusions}

The generation of nanoparticles from NiTi-alloy target by laser ablation in distilled water and ethanol has been demonstrated. The size of the nanoparticles ranged between 50 and $1000 \mathrm{~nm}$ and showed slight fluence dependence. SEM and EDX analyses have shown that spherical particles with different compositions were created. The non-uniform elemental composition observed in case of water may be attributed to the Ti oxidation. Unfortunately our system was unable to give reliable information on the oxygen abundance of the samples, however it is plausible that the differences observed in the case of water and ethanol can be attributed to the different oxidizing effect of the components formed during laser irradiation. The elemental separation already starts at the surface of the ablated nitinol, leading to ejection of pure $\mathrm{Ni}$ or $\mathrm{Ti}$ nanoparicles besides the mixed ones. The results suggest that the proper choice of the laser parameters and the applied liquids gives this technique flexibility in controlling the properties of nanoparticles. With increasing the fluence some of the larger particles showed a core-shell composition: the core always consisted of $\mathrm{Ni}$, while titanium was accumulated in the outer region. Further research and analysis of the particles obtained is needed for a better understanding of particle formation with the experiment parameters used.

\section{Acknowledgments}

This work was partially supported by the European Union and co-financed by the European Social Fund through projects 'Impulse lasers for use in materials science and biophotonics' (grant no. TÁMOP-4.2.2.A-11/1/KONV2012-0060), and 'Biological and environmental responses initiated by new functional materials' (grant no. TÁMOP4.2.2.A-11/1/KONV-2012-0047).

\section{References}

[1] R. M. Tilaki, A. Iraji-Zad, and S. M. Mahdavi, Appl. Phys. A 84, (2006) 215.

[2] S. Barcikowski, N. Bärsch, M. Hustedt, R. Sattari, A. Ostendorf: Proc. ICALEO 23, (2005) 375.

[3] M. H. Wu, R. Mu, A. Ueda, D. O. Henderson: Mat. Res. Soc. Symp. Proc. 780, (2003) Y3.2/1.

[4] J. Koch, A. von Bohlen, R. Hergenröder, K. Niemax: J. Anal. At. Spectrom. 19, (2004) 267.

[5] A.Hahn, S. Barcikowski, B. N. Chichkov, J. of Laser Micro/Nanoengineering Vol. 3, No. 2, (2008)

[6] H. Zeng, X.-W. Du, S. C. Singh, S.A. Kulinich, S Yang, J. He, W. Cai, Adv. Funct. Mater. 22, (2012) 1333.

[7] S. Barcikowski, M. Hustedt, B. Chichkov, Polimery 53 (2008), 657.

[8] J. Sun, S. L. Hu, X. W. Du, Y. W. Lei, L. Jiang, Appl. Phys. Lett. 89 (2006) Article ID 183115.

[9] M. Chakif, Shape Memory and Superelastic Technologies (SMST), (2013)

[10] W. Qi, L. Luo, H-S. Qian, G. Ouyang, K. Kar Nanda, S. O. Obare Journal of Nanomaterials Vol. 2012 (2012), Article ID 526923

[11] N.G. Semaltianos, S. Logothetidis, N. Frangis, I Tsiaoussis, W. Perrie, G. Dearden, K.G. Watkins, Chem. Phys. Lett. 496, (2010) 113.

[12] M. Boutinguiza, J. del Val, A. Riveiro, F. Lusquiños, F. Quintero, R. Comesaña, J. Pou, Physics Procedia 41, (2013) 787.

[13] G.S. Firstov, R.G. Vitchev1, H. Kumar, B. Blanpain, J. Van Humbeeck, Biomaterials 23, (2002) 4863.

(Received: June 16, 2014, Accepted: March 3, 2015) 